\title{
Dynamical Classification of Trans-Neptunian Objects Detected by the Dark Energy Survey
}

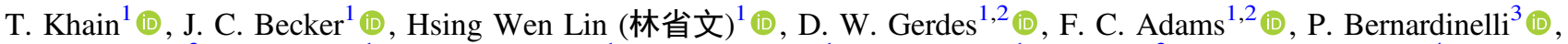
G. M. Bernstein ${ }^{3}$, K. Franson ${ }^{1}$ (1), L. Markwardt ${ }^{1}$ (D) S. Hamilton ${ }^{1}$ (1), K. Napier ${ }^{1}$, M. Sako ${ }^{3}$ (D) T. M. C. Abbott ${ }^{4}$, S. Avila ${ }^{5}$, E. Bertin ${ }^{6,7}$, D. Brooks ${ }^{8}$ (), E. Buckley-Geer ${ }^{9}$, D. L. Burke ${ }^{10,11}$ (1), A. Carnero Rosel1 ${ }^{12,13}$, M. Carrasco Kind ${ }^{14,15}$ (1), J. Carretero ${ }^{16}$, L. N. da Costa $^{13,17}$, J. De Vicente ${ }^{12}$, S. Desai ${ }^{18}$, H. T. Diehl9 ${ }^{9}$, P. Doel ${ }^{8}$, B. Flaugher ${ }^{9}$, J. Frieman ${ }^{9,19}$, J. García-Bellido ${ }^{5}$ (i), E. Gaztanaga ${ }^{20,21}$, D. Gruen ${ }^{10,11,22}$ (i), R. A. Gruendl ${ }^{14,15}$ (i), J. Gschwend ${ }^{13,17}$, G. Gutierrez ${ }^{9}$, D. L. Hollowood ${ }^{23}$ (10), K. Honscheid ${ }^{24,25}$, D. J. James ${ }^{26}$, N. Kuropatkin ${ }^{9}$, M. A. G. Maia ${ }^{13,17}$, J. L. Marshall ${ }^{27}$ (10, F. Menanteau ${ }^{14,15}$, C. J. Miller ${ }^{1,2}$,

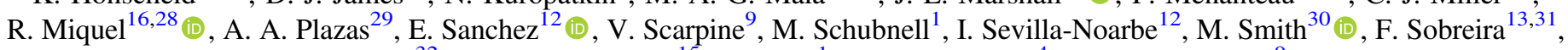
E. Suchyta ${ }^{32}$, M. E. C. Swanson ${ }^{15}$, G. Tarle ${ }^{1}$ (D), A. R. Walker ${ }^{4}$ (10), and W. Wester ${ }^{9}$

The Dark Energy Survey Collaboration

${ }^{1}$ Department of Physics, University of Michigan, Ann Arbor, MI 48109, USA; talikh@umich.edu

${ }^{2}$ Department of Astronomy, University of Michigan, Ann Arbor, MI 48109, USA

${ }^{3}$ Department of Physics and Astronomy, University of Pennsylvania, Philadelphia, PA 19104, USA

${ }^{4}$ Cerro Tololo Inter-American Observatory, National Optical Astronomy Observatory, Casilla 603, La Serena, Chile

${ }^{5}$ Instituto de Fisica Teorica UAM/CSIC, Universidad Autonoma de Madrid, E-28049 Madrid, Spain ${ }^{6}$ CNRS, UMR 7095, Institut d'Astrophysique de Paris, F-75014, Paris, France

${ }^{7}$ Sorbonne Universités, UPMC Univ Paris 06, UMR 7095, Institut d'Astrophysique de Paris, F-75014, Paris, France

${ }^{8}$ Department of Physics \& Astronomy, University College London, Gower Street, London, WC1E 6BT, UK

${ }_{9}^{9}$ Fermi National Accelerator Laboratory, P.O. Box 500, Batavia, IL 60510, USA

${ }^{10}$ Kavli Institute for Particle Astrophysics \& Cosmology, P.O. Box 2450, Stanford University, Stanford, CA 94305, USA

${ }^{11}$ SLAC National Accelerator Laboratory, Menlo Park, CA 94025, USA

${ }^{12}$ Centro de Investigaciones Energéticas, Medioambientales y Tecnológicas (CIEMAT), Madrid, Spain

${ }^{13}$ Laboratório Interinstitucional de e-Astronomia-LIneA, Rua Gal. José Cristino 77, Rio de Janeiro, RJ-20921-400, Brazil

${ }^{14}$ Department of Astronomy, University of Illinois at Urbana-Champaign, 1002 W. Green Street, Urbana, IL 61801, USA

${ }^{15}$ National Center for Supercomputing Applications, 1205 West Clark St., Urbana, IL 61801, USA

${ }^{16}$ Institut de Física d'Altes Energies (IFAE), The Barcelona Institute of Science and Technology, Campus UAB, E-08193 Bellaterra (Barcelona), Spain

${ }^{17}$ Observatório Nacional, Rua Gal. José Cristino 77, Rio de Janeiro, RJ-20921-400, Brazil

${ }^{18}$ Department of Physics, IIT Hyderabad, Kandi, Telangana 502285, India

${ }^{19}$ Kavli Institute for Cosmological Physics, University of Chicago, Chicago, IL 60637, USA

${ }^{20}$ Institut d'Estudis Espacials de Catalunya (IEEC), E-08034 Barcelona, Spain

${ }^{21}$ Institute of Space Sciences (ICE, CSIC), Campus UAB, Carrer de Can Magrans, s/n, 08193 E-Barcelona, Spain

${ }^{22}$ Department of Physics, Stanford University, 382 Via Pueblo Mall, Stanford, CA 94305, USA ${ }^{23}$ Santa Cruz Institute for Particle Physics, Santa Cruz, CA 95064, USA

${ }^{24}$ Center for Cosmology and Astro-Particle Physics, The Ohio State University, Columbus, OH 43210, USA

${ }^{25}$ Department of Physics, The Ohio State University, Columbus, OH 43210, USA

${ }^{26}$ Center for Astrophysics | Harvard \& Smithsonian, 60 Garden Street, Cambridge, MA 02138, USA

${ }^{27}$ George P. and Cynthia Woods Mitchell Institute for Fundamental Physics and Astronomy, and Department of Physics and Astronomy, Texas A\& M University, College Station, TX 77843, USA

${ }^{28}$ Institució Catalana de Recerca i Estudis Avançats, E-08010 Barcelona, Spain

${ }^{29}$ Department of Astrophysical Sciences, Princeton University, Peyton Hall, Princeton, NJ 08544, USA

${ }^{30}$ School of Physics and Astronomy, University of Southampton, Southampton, SO17 1BJ, UK

${ }^{31}$ Instituto de Física Gleb Wataghin, Universidade Estadual de Campinas, 13083-859, Campinas, SP, Brazil

${ }^{32}$ Computer Science and Mathematics Division, Oak Ridge National Laboratory, Oak Ridge, TN 37831, USA

Received 2019 May 31; revised 2020 January 10; accepted 2020 January 15; published 2020 February 28

\begin{abstract}
The outer solar system contains a large number of small bodies (known as trans-Neptunian objects or TNOs) that exhibit diverse types of dynamical behavior. The classification of bodies in this distant region into dynamical classes - subpopulations that experience similar orbital evolution-aids in our understanding of the structure and formation of the solar system. In this work, we propose an updated dynamical classification scheme for the outer solar system. This approach includes the construction of a new (automated) method for identifying mean motion resonances. We apply this algorithm to the current data set of TNOs observed by the Dark Energy Survey (DES) and present a working classification for all of the DES TNOs detected to date. Our classification scheme yields 1 inner centaur, 19 outer centaurs, 21 scattering disk objects, 47 detached TNOs, 48 securely resonant objects, 7 resonant candidates, and 97 classical belt objects. Among the scattering and detached objects, we detect 8 TNOs with semimajor axes greater than 150 au.
\end{abstract}

Unified Astronomy Thesaurus concepts: Kuiper belt (893); Orbital evolution (1178); N-body simulations (1083) Supporting material: machine-readable table 


\section{Introduction}

Our solar system harbors a large collection of small icy bodies that orbit the Sun beyond Neptune. In the past two decades, the number of these trans-Neptunian objects (TNOs) that has been discovered has grown to thousands. As these objects are believed to be primordial tracers of the early solar system, the characterization of the trans-Neptunian population is vital for understanding and testing theoretical models of solar system formation. For example, in one class of theories collectively known as the Nice Model (Tsiganis et al. 2005; Nesvorný 2011; Batygin et al. 2012), the starting orbits of the giant planets are different from those of the present epoch. Such models predict sizes and distributions of the different subpopulations of TNOs in the Kuiper Belt due to the orbital migration of the larger planets to their current locations.

Over the past decades, a number of surveys intended to study the outer solar system have significantly increased the population of known TNOs (e.g., Trujillo et al. 2001; Schwamb et al. 2010; Petit et al. 2011; Adams et al. 2014; Bannister et al. 2018), allowing these theories to be tested. Today, the growing number of observed objects combined with the development of survey simulators (Lawler et al. 2018; S. J. Hamilton \& DES Collaboration 2020, in preparation) allows for detailed comparisons of the observed and predicted populations (Volk et al. 2016, 2018) as expected within single modern surveys.

The TNOs themselves can be characterized in a variety of ways, including their size, color, and composition. These physical properties of the objects, however, are often difficult to observe. Fortunately, the orbits of the objects can provide insight into the structure and dynamical history of this distant region. By categorizing the TNOs based on their dynamical behaviors, we can extract information about the various subpopulations of the outer solar system. The primary works that laid out this type of dynamical classification scheme are those of Elliot et al. (2005) and Gladman et al. (2008); the major dynamical classes of the Kuiper Belt include the Neptune-resonant objects, centaurs, scattering disk objects (SDO), detached TNOs, and more (see below).

One of the surveys that has led to the discovery of these Kuiper Belt objects is the Dark Energy Survey (DES; Dark Energy Survey Collaboration et al. 2016), a nominal five year baseline optical survey intended primarily for cosmological purposes. DES used the Dark Energy Camera (DECam, Flaugher et al. 2015) on the $4 \mathrm{~m}$ Blanco telescope at the Cerro Tololo Inter-American Observatory in Chile. Its survey area subtended a total of 5000 square degrees of sky, which was tiled with two survey modes: the Wide Survey, which imaged the full survey area roughly twice per year to a limiting magnitude of $r \sim 23.8$ mag for single epoch exposures in each of the griz $Y$ bands; and the Supernova Survey (Bernstein et al. 2012), which consisted of 30 square degrees spread over 10 regions, each of which were imaged roughly weekly in the griz bands.

In a partial search of its first four years of data, DES has detected over 200 TNOs (and counting). The discoveries so far include Neptune trojans (Gerdes et al. 2016; Lin et al. 2019), a dwarf planet candidate (Gerdes et al. 2017), two members of a potentially associated triplet family (Khain et al. 2018), and a high-inclination extreme TNO (Becker et al. 2018), with further publications detailing the results of additional analysis to come. Now that the current DES data set has grown to this

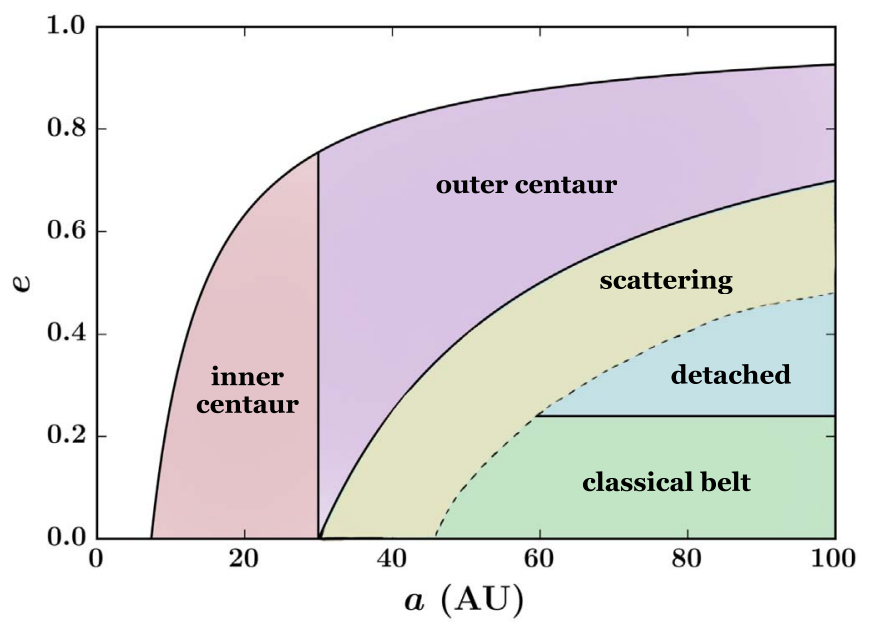

Figure 1. Dynamical classes of the outer solar system. The black solid curves correspond to constant perihelion distances, with $q=7.35$ au and $q=30$ au (top to bottom). The inner centaurs (red region) have orbital periods less than Neptune's. The outer centaurs (purple) have orbits with perihelion distances below Neptune's orbit, but with semimajor axes outside the giant planet region. The scattering population (SDOs, scattering disk objects) mostly lies along the $q=30$ au curve and is shown in yellow. The classical belt (green region) and the detached objects (blue region) are removed from the Neptune scattering region, with the higher eccentricity detached TNOs above the classical belt. A companion plot with the DES TNOs on this phase plane is found in Figure 8.

substantial size, it is of great interest to study the dynamical properties of this TNO population.

In this work, we present the dynamical classification of 240 TNOs detected by the DES. Although the present application is to this particular set of TNOs, the classification scheme developed herein can be used more broadly. In Section 2, we lay out the different categories of TNOs and our classification algorithm, which differs somewhat from that of Gladman et al. (2008). In addition, we outline our newly developed resonancefinding method that allows for an automated resonance search without visual inspection. In Section 3, we apply this algorithm to the object sample and present the classification of the known DES TNOs. We discuss our results and their implications for future work in Section 4.

\section{Classification Method}

In this work, we apply the classification scheme of Gladman et al. (2008) with a few changes that reflect the development of the field in the last decade. The categories of objects and the definitions we adapt are described below and are visually represented in Figure 1. As with any classification scheme, a few of the category boundaries are rather arbitrary, as some of these dynamical properties lie on a spectrum. Deviations from Gladman et al. (2008) are denoted with an asterisk* .

Jupiter-coupled object. Jupiter-coupled objects are defined through the Tisserand parameter $T_{\mathrm{J}}$ with respect to Jupiter,

$$
T_{\mathrm{J}}=\frac{a_{\mathrm{J}}}{a}+2 \sqrt{\frac{a}{a_{\mathrm{J}}}\left(1-e^{2}\right)} \cos i,
$$

where $a_{\mathrm{J}}$ is the semimajor axis of Jupiter, and $a, e$, and $i$ are the semimajor axis, eccentricity, and inclination of the object, respectively. Objects with $T_{\mathrm{J}}<3.05$ and perihelion distances below $q<7.35 \mathrm{au}$ are considered to be Jupiter-coupled objects. 
Since the current DES sample does not contain any objects which exhibit cometary dynamics, we drop this category in future discussion of the classification results.

Centaur*. Centaurs are objects that experience strong interactions with the giant planets. In this work, we propose to separate this class into two: inner centaurs and outer centaurs. Inner centaurs (the traditional centaurs described in Gladman et al. 2008) are objects with semimajor axes smaller than Neptune's ( $a<a_{\mathrm{N}} \approx 30 \mathrm{au}$ ). We define outer centaurs to be objects with perihelion distances shorter than Neptune's semimajor axis $\left(q<a_{\mathrm{N}}\right)$, but semimajor axes larger than Neptune's semimajor axis $\left(a>a_{\mathrm{N}}\right)$.

Although both types of centaurs spend time within the giant planet region, the frequency of interactions with the planets differs for each class. The inner centaurs may experience strong interactions with the giant planets at most points on their orbit, while the outer centaurs are affected once an orbit, during perihelion crossing; moreover, the orbital period of an outer centaur is longer than of an inner centaur, resulting in fewer interactions per unit time. This distinction highlights the difference in the instability timescale: the outer centaurs are longer-lived objects than the short lifetime inner centaurs (Tiscareno \& Malhotra 2003; Horner et al. 2004). By this classification, a traditional centaur such as Chiron (Kowal et al. 1979) falls into the inner centaur category, while longer-period objects with high eccentricity such as Drac (Gladman et al. 2009) or Niku (Chen et al. 2016) are deemed outer centaurs.

An example of the dynamics of inner and outer centaurs from the DES set is shown in Figure 2.

Oort cloud object. Objects in the Oort cloud are defined to have semimajor axes $a>2000$ au. Due to their large orbits, these bodies are most likely affected by galactic tides and passing stars. The present DES sample does not contain any objects in this class.

Resonant object. The outer solar system consists of a large number of TNOs in mean motion resonances with Neptune. In order to be in a Neptune mean motion resonance, a TNO must be near an integer period ratio with Neptune's period, and must have a librating resonance argument of the form

$$
\phi=p \lambda_{\mathrm{N}}+q \lambda+r \varpi_{\mathrm{N}}+s \varpi,
$$

where $p, q, r$, and $s$ are integers that satisfy the d'Alembert relation, $p+q+r+s=0$. Here, $\lambda=\Omega+\omega+M$ is the mean longitude, $\varpi=\Omega+\omega$ is the longitude of perihelion, the subscript $N$ refers to Neptune's orbital elements, and the nonsubscripted variables refer to the TNO. Such a resonance is then referred to as a $p: q$ resonance, the ratio of Neptune's orbital period to that of the TNO. In this work, we only consider the eccentricity-type resonances given by Equation (2), as was done in Gladman et al. (2008). In theory, TNOs could also experience inclination-type resonances, which include independent $\Omega$ and $\Omega_{\mathrm{N}}$ terms. Since these are a higher order effect, we leave the study of inclination-type resonances for future work.

An example of a resonant TNO from the DES data is shown in the left column of Figure 3. Note the constant behavior of the semimajor axis in the top panel; the inset demonstrates the librating resonance argument corresponding to the 2:7 commensurability.

$S D O^{*}$. SDOs are objects that are currently scattering off of Neptune and experience rapid and significant variations in their semimajor axis evolution as a result. Unlike the outer centaurs, the orbits of the scattering objects lie fully outside the giant planet region, and thus SDOs experience rather weak interactions with Neptune. Consistent with the Gladman et al. (2008) definition, we define a scattering object as one whose semimajor axis changes by more than a few astronomical units with respect to its initial value, $a_{0}$, over the integration time (10 Myr for objects with $a<100 \mathrm{au}$, and $100 \mathrm{Myr}$ for objects with $a>100 \mathrm{au}$ ). To ensure that this definition scales well as we consider longer-period objects, our criterion for scattering is as follows:

$$
\frac{\Delta a}{a}>0.0375
$$

where

$$
\frac{\Delta a}{a}=\frac{\max \left(a(t)-a_{0}\right)}{a_{0}}
$$

is the maximum variation in semimajor axis over the integration time. The choice of the exact value of variation allowed before an object becomes an SDO is somewhat arbitrary, but it must be large enough that periodic variations of orbital elements do not falsely classify an object as scattering. Here we use the value of 0.0375 , as it corresponds to the accepted change of 1.5 au for a typical classical belt object at $a=40$ au (Gladman et al. 2008). Previous works have also used $\Delta a / a<0.05$ (Volk \& Malhotra 2017) and $1.5 \mathrm{au}$ (Morbidelli et al. 2004). An example of the dynamics of a scattering object from the DES set is shown in the left column of Figure 4. Note the significant change in the semimajor axis over the short $10 \mathrm{Myr}$ integration time, as well as the proximity of the perihelion distance to Neptune's orbit at $30 \mathrm{au}$.

Detached object. Detached TNOs are objects whose dynamics are decoupled from Neptune's influence. Generally, these are TNOs with large perihelion distances; following Gladman et al. (2008), we define nonscattering and nonresonant TNOs with eccentricities $e>0.24$ to be detached. Most of these objects are found beyond the 1:2 resonance with Neptune $(a>47.7 \mathrm{au}$ ). An example of a detached TNO is shown in the right column of Figure 4. Note the large perihelion distance and the resulting undisturbed semimajor axis evolution.

Classical belt object. The classical belt, then, is composed of nonscattering TNOs with eccentricities $e<0.24$. An example of such an object is shown in the right column of Figure 3.

A visual representation of these dynamical regimes on the semimajor axis-eccentricity plane can be found in Figure 1. A companion plot that shows the DES TNOs in each class and a detailed discussion of these results is found in Section 3.

Given the definitions above, we begin by checking each object in our sample for resonant behavior. If nonresonant, we proceed to classify its dynamics into one of the remaining classes.

Although it may be possible to determine whether an object fits into one of the above categories just by considering its present day orbit, we cannot fully classify the objects without understanding their orbital evolution. The two categories that require this knowledge are the resonant and scattering classes; without running numerical simulations that model the outer solar system, we cannot classify such objects.

Using the categories outlined above, we present our algorithm for TNO classification below. 

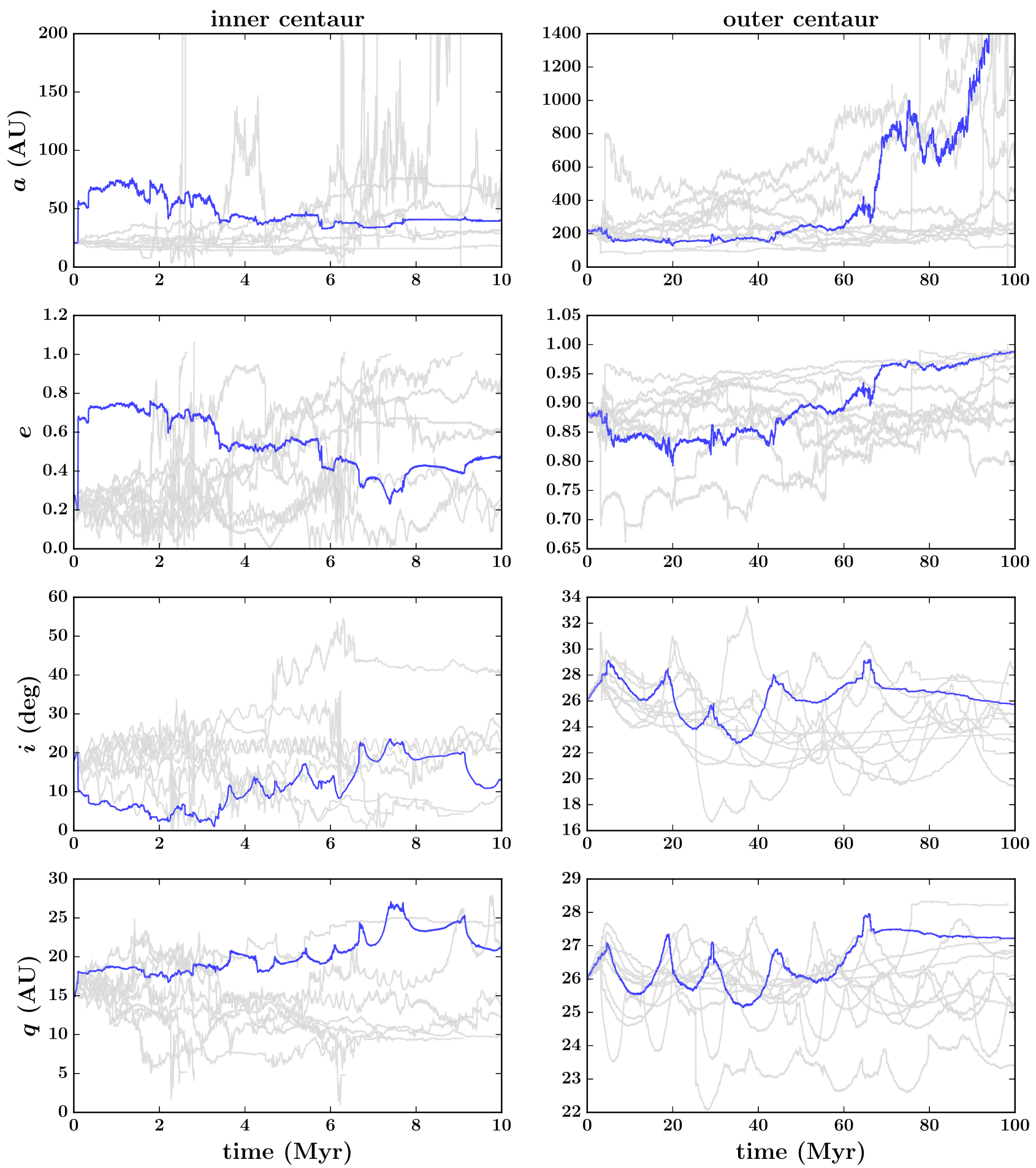

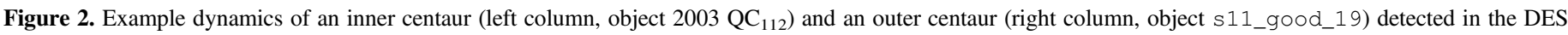

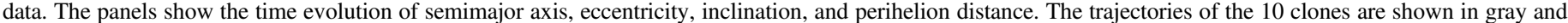
the best-fit trajectory is in blue. Note the short perihelion distance of the two centaurs.

1. From observations, determine the best-fit orbital elements and the associated covariance matrix for each object. In this work, we use the fitting algorithm from Bernstein \& Khushalani (2000).

2. Generate 10 clones of each TNO by drawing from a sixdimensional Gaussian distribution, where the best-fit orbit is the mean and the covariance matrix represents the uncertainties.
3. Run an $N$-body integration of the 10 clones and the bestfit orbit. In order to properly compare classifications for different objects, it is best if the dynamical behavior is evaluated for approximately the same number of orbital periods. For this reason, we run $10 \mathrm{Myr}$ integrations for objects with $a<100$ au and $100 \mathrm{Myr}$ integrations for objects with $a>100 \mathrm{au}$. The threshold of $100 \mathrm{au}$ is an arbitrary choice, but the integrations must be extended for 

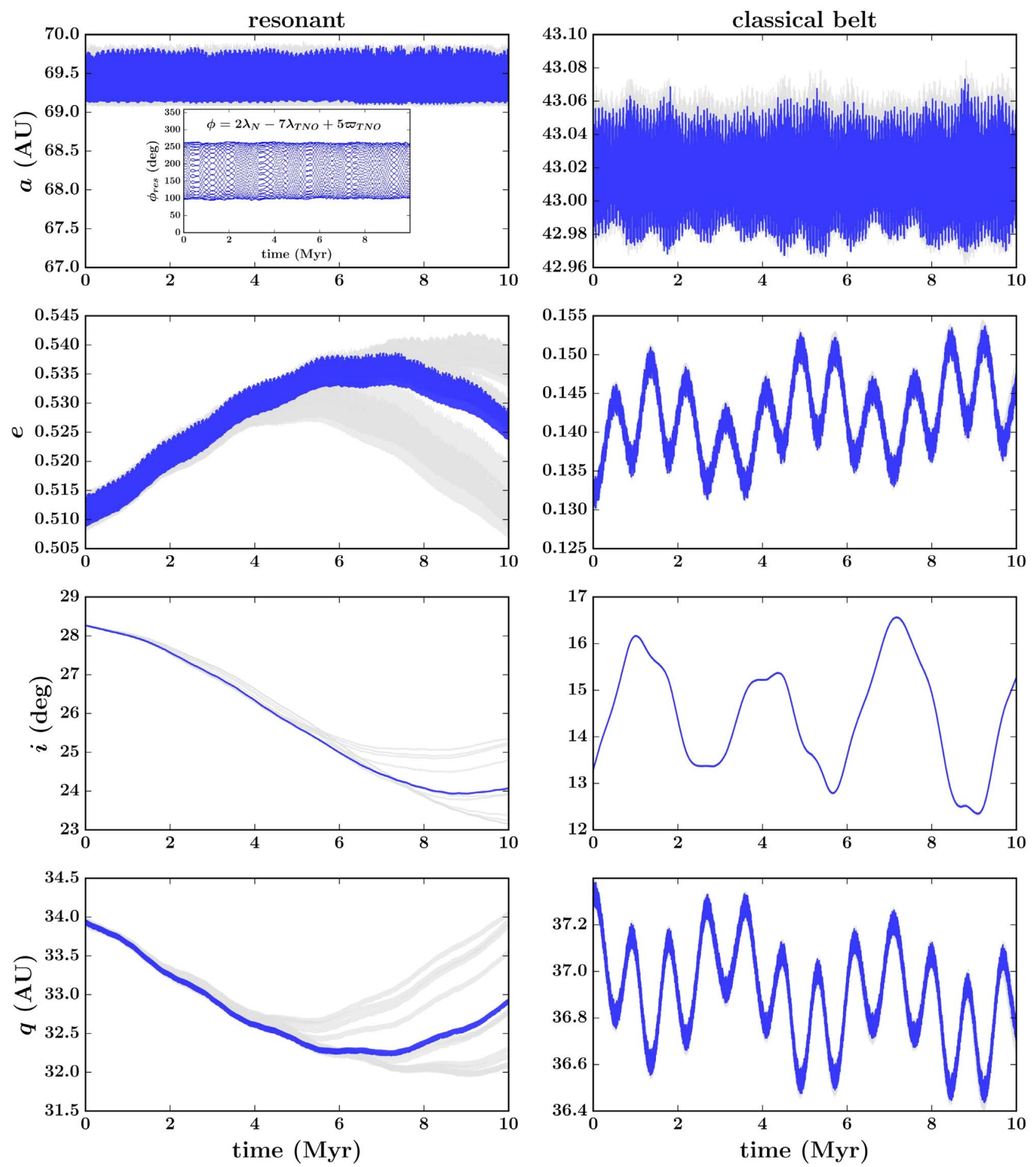

Figure 3. Example dynamics of a resonant object (left column, object s12_good_5) and a classical belt object (right column, object $2013 \mathrm{RP}_{98}$ ) detected in the DES data. The panels show the time evolution of semimajor axis, eccentricity, inclination, and perihelion distance. The trajectories of the 10 clones are shown in gray and the best-fit trajectory is in blue. The inset in the top left panel displays the time evolution of the resonant argument corresponding to the 2:7 resonance of the TNO; note that the behavior of this angle is bounded (librating), indicating that this TNO is in fact in resonance for the full integration time.

longer-period objects as it takes more time to evaluate the dynamics. We use the $N$-body code mercury 6 with a hybrid symplectic and Bulirsch-Stoer (B-S) integrator and a time step of 20 days. In each integration, we include the TNO and its clones as test particles, as well as the four giant planets as active bodies (Jupiter, Saturn, Uranus, and Neptune). We integrate the orbital elements for each TNO to a common epoch before beginning the simulations; in this work, time zero corresponds to the date 2019 May 4th.

4. Dynamically classify the objects based on the output of the simulations. The TNOs are grouped into the Jupitercoupled object, inner centaur, outer centaur, Oort cloud, detached, and classical belt classes based on the current 

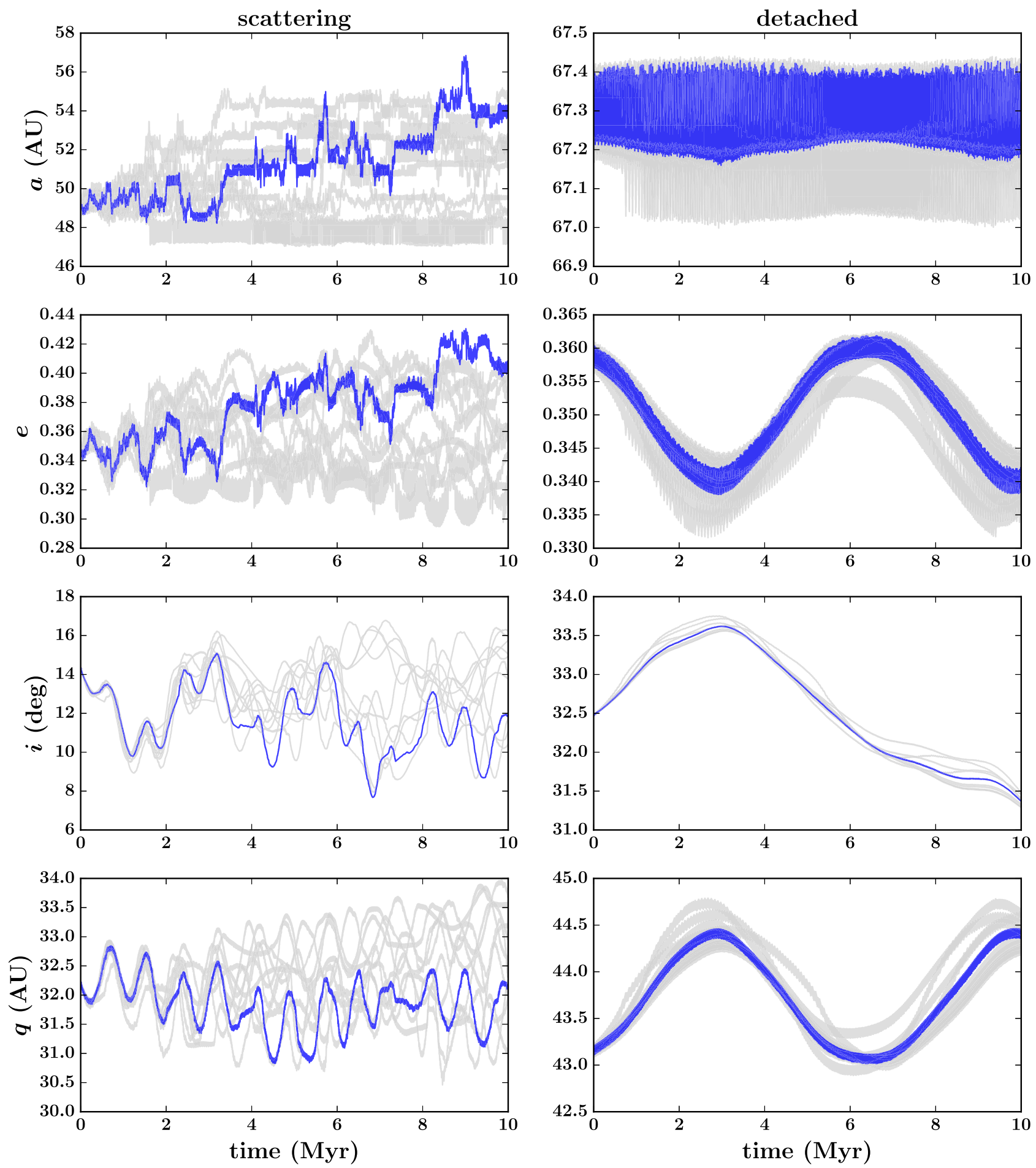

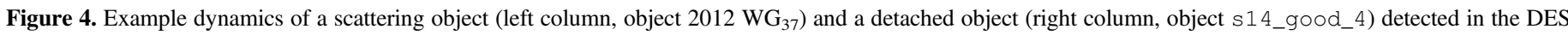

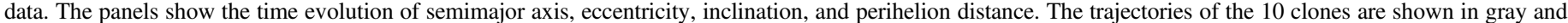

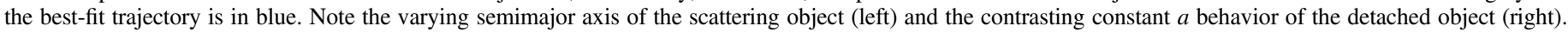

day best-fit orbit. The resonant and scattering classifications are based on the time evolution of the 10 clones. In particular, we consider TNOs with more than five clones that experience scattering behavior (as defined above) to be scattering objects. The resonant classification is more strict; only objects that are resonant for greater than $95 \%$ of the time, averaging over the 10 clones, are considered to be resonant objects. Additional details regarding the resonance classification can be found in Section 3.1.
5. Check if there are objects with insecure classifications. Such TNOs generally have clones with orbits that are different enough to cause them to experience disparate dynamical evolution. For example, in our data, we found that a handful of TNOs would have a couple of scattering clones, but the rest of their clones would be detached. In this situation, we extend the integration time to $100 \mathrm{Myr}$ to enable a more secure classification. If the classification remains insecure, we sort the object into a category as 

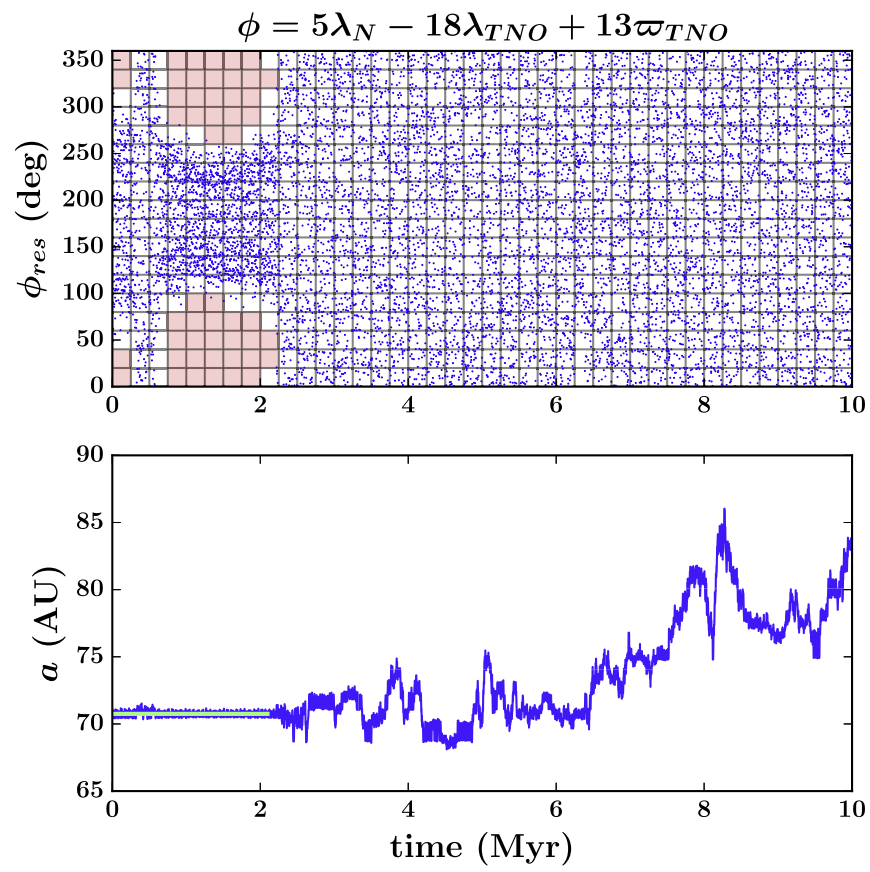

Figure 5. Demonstration of the automated resonance identification algorithm The top panel shows the time evolution of the resonance argument $\phi$ in small blue markers. The grid guides the search for low-point-density rectangles, which are shaded in light red. The bottom panel shows the corresponding semimajor axis evolution, with regions of constant $a$ highlighted in green. Note that this figure demonstrates a likely nonresonant object; this particular clone only spends a small portion of the integration time in resonance.

delineated in step 4, and leave the question of secure classification for future work, once higher precision orbits are acquired.

As can be seen from the dynamical class definitions above, it is straightforward to automatically separate the TNOs into the Jupiter-coupled object, inner centaur, outer centaur, Oort cloud, scattering, detached, and classical belt categories. The tricky step of the process is the resonance classification. To classify an object as resonant, it must not only be near an integer period ratio with Neptune, but we must identify a librating resonance angle. Often in the literature, this analysis is done by hand. Since the DES data set contains hundreds of objects, this becomes significantly time intensive. In addition, since each period ratio has a large number of resonance arguments associated with it (i.e., for each $p, q$ pair, there are many $r, s$ pairs that satisfy $p+q+r+s=0$ ), it is difficult to conclude with certainty that an object is nonresonant.

In the following subsection, then, we describe the resonance identification algorithm we have developed to address these challenges. The main idea behind the algorithm lies in plotting the time evolution of many potential resonance arguments, and searching for regions of libration by identifying low-pointdensity regions in the plot. By applying this strategy, we are able to successfully identify a number of resonant objects, some of which are in rather high order resonances with Neptune.

\subsection{Resonance Identification}

In this subsection, we describe the resonance identification process. The input for this algorithm are the simulation results for the 10 clones of the TNO; each clone is studied
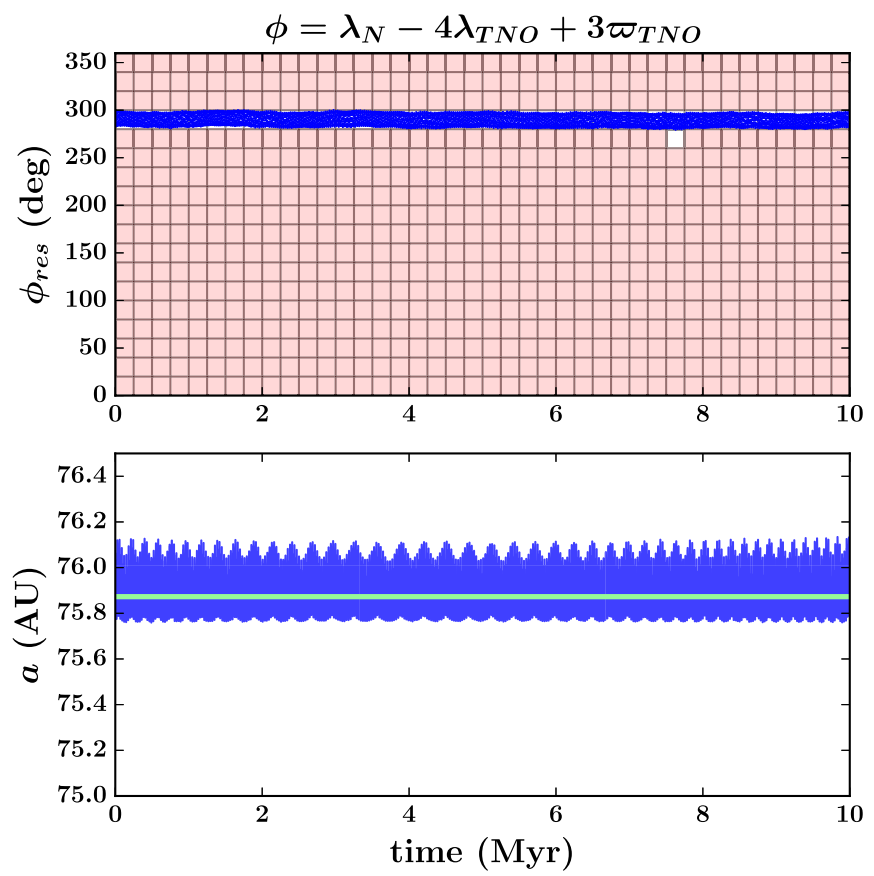

Figure 6. Demonstration of the automated resonance identification algorithm. The top panel shows the time evolution of the resonance argument $\phi$ in small blue markers. The grid guides the search for low-point-density rectangles, which are shaded in light red. The bottom panel shows the corresponding semimajor axis evolution, with regions of constant $a$ highlighted in green. In contrast to Figure 5, this clone is in resonance for the full integration time. The large number of shaded grid squares indicate the clearly bounded resonance angle evolution.

individually, as described below. A sample of this procedure is demonstrated in Figures 5 and 6.

1. Divide the total integration time into shorter time intervals. Since the algorithm is based on a point-density analysis, we have found that it is best if each interval contains $\sim 5000$ data points. In our $10 \mathrm{Myr}$ integrations, this corresponds to $5 \mathrm{Myr}$ intervals, and $50 \mathrm{Myr}$ intervals in our $100 \mathrm{Myr}$ integrations. This coarse subdivision allows us to identify regions of constant semimajor axis; as described below, we break up these time intervals further in later steps of the process.

2. Average over the semimajor axis evolution in each interval, and compute the corresponding averaged period ratio with Neptune, $R_{\mathrm{av}}$.

3. If the average period ratios in neighboring intervals have similar values, connect the time intervals. In our analysis, we connect these intervals if the period ratios differ by less than 0.01. In the steps that follow, we will search for resonances in each of these connected intervals.

4. Recall that the resonance argument is of the form

$$
\phi=p \lambda_{\mathrm{N}}+q \lambda+r \varpi_{\mathrm{N}}+s \varpi,
$$

where $p, q, r$, and $s$ are integers that satisfy $p+$ $q+r+s=0$. For each interval, consider a range of $p$ : $q$ resonances that span the period ratio range of $\left(R_{\mathrm{av}}-\right.$ resonance width, $R_{\mathrm{av}}+$ resonance width). In our analysis, we use a resonance width value of 0.2 , which corresponds to a range of about 7 au at a semimajor axis of $39 \mathrm{au}$. Note that this purposefully overestimates the resonant width to ensure that all possible resonances are considered; realistic calculations of the semimajor axis 
width for Neptune resonances can be found in Wang \& Malhotra (2017) and Lan \& Malhotra (2019).

5. Identify the first $p: q$ resonance within the period ratio range. Here, a decision needs to be made regarding the order of the resonances considered. In our analysis, we check all resonance arguments with $p,|q| \in[1,26]$, and $r, s \in[-25,24]$.

6. Fix the first pair of $r$ and $s$ coefficients.

7. Next, overlay a fine grid on the plot of $\phi$ versus time over one time interval. We use a grid of 18 horizontal lines, as $\phi \in\left(0^{\circ}, 360^{\circ}\right)$, and 20 vertical lines for every 5000 points (see the top panel of Figures 5 and 6).

8. Run over the grid, counting the number of points in each grid square. Flag grid squares with few points (for the parameters specified above, we flag squares with one or zero points). In Figures 5 and 6, flagged squares are shaded in light red. Next, impose additional restrictions on the grid to correctly identify resonances; we require that there must be at least two flagged squares per column, or at least two adjacent flagged squares per row, and require a total number of flagged squares to exceed a set threshold. These additional conditions help discard false positives, and can be adjusted depending on the data one is working with.

9. Repeat steps 6-8 for each pair of $r, s$ coefficients which satisfy the resonance relationship for the chosen $p: q$ resonance. Once all $r, s$ pairs have been cycled through, identify the best $r, s$ pair by choosing the one with the largest number of flagged grid squares.

10. Repeat steps 5-9 for the entire set of $p: q$ pairs.

11. Repeat steps 1-10 for each clone of the TNO. Compute the fraction of time spent in resonance by each clone, and average over all clones to find the resonance percentage for the TNO.

In this process, then, we parse the simulation data on a variety of timescales. First, we identify the regions of constant semimajor axis on long time intervals, and then check the resonance argument libration precisely on a fine subdivided grid. To achieve the best results, the exact length of these intervals should scale with the orbital period of the object one is studying.

After applying this algorithm, a decision needs to be made regarding the percentage threshold at which a TNO is considered to be truly resonant. In our analysis, we define objects that are resonant for greater than $95 \%$ of the time to be resonant, and objects that are resonant for greater than $50 \%$ of the time to be resonant candidates. The application of this procedure to the current DES TNO sample and the analysis of the results is described in the following section.

\section{Classification of DES TNOs}

We apply the algorithm described in Section 2 to the currently available data set of DES TNOs. The sample does not contain any Jupiter-coupled objects or Oort cloud objects, but all other dynamical classes are represented. We find 1 inner centaur, 19 outer centaurs, 21 SDOs, 47 detached TNOs, 48 securely resonant objects, 7 resonant candidates, and 97 classical belt objects. The classifications for specific objects and their barycentric orbital elements are reported in Table 1.

A visual summary of these results is shown in the bar plot in Figure 7. The classical belt population dominates the data set,

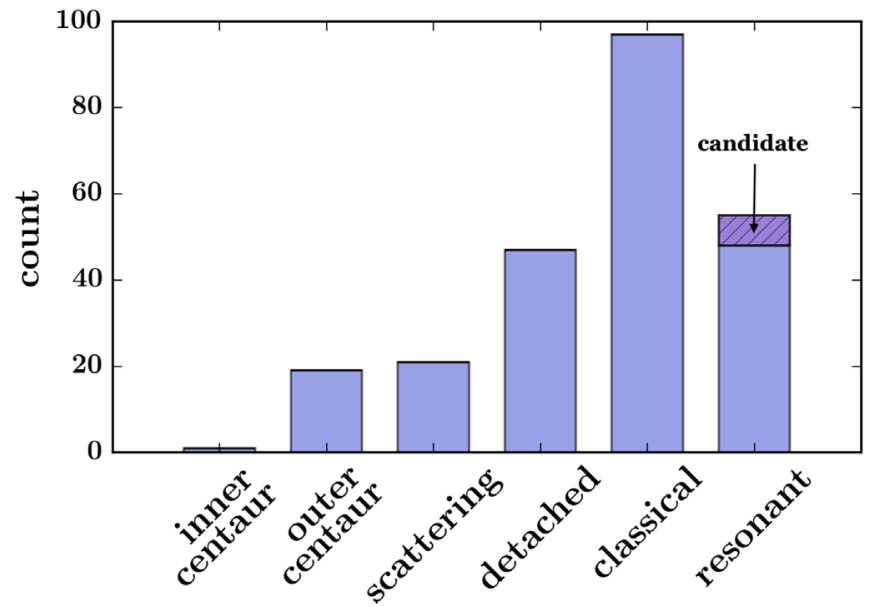

Figure 7. Summary plot of the dynamical classification of the DES TNOs, showing the relative abundance of each category out of the 240 total classified objects. Most of the objects detected in the data are members of the classical belt, but there are a number of both detached and securely resonant objects as well. Resonant objects that could not be securely identified are marked as candidates.

but there is a significant number of detached and resonant TNOs as well. The resonant bar consists of two parts; the blue represents the securely resonant objects, while the purple shows the resonant candidates.

This data is further visualized on the semimajor axiseccentricity plane in Figure 8. The black solid curves correspond to constant perihelion distances, with $q=7.35 \mathrm{au}$ and $q=30 \mathrm{au}$, from top to bottom. A companion plot that presents the regions of each dynamical class can be found in Figure 1; the colors of the regions correspond to the marker colors in Figure 8.

In Figure 8, the current day best fit $(a, e)$ of each TNO is plotted with a colored marker that corresponds to its dynamical class. The inner centaurs, in red, are found in the giant planet region, with semimajor axes below $a_{\mathrm{N}}=30 \mathrm{au}$, and the outer centaurs, in purple, cross Neptune's orbit, with $q<30$ au and $a>30 \mathrm{au}$. Most of the other objects are found near the $q=30$ au curve, as it is easier to observe short perihelion TNOs. There are a few exceptions; most notably, a detached TNO in blue with $a=105$ au and $q=50$ au (s17_good_0).

The population of objects denoted with green markers at low eccentricity constitute the classical belt. These TNOs are dynamically cold (undergo only minimal orbital evolution) as they do not experience strong interactions with Neptune. Their perihelion detachment is evident in the inset plot, which zooms in on the $a \in[30,60], e \in[0,0.5]$ region, and demonstrates that the classical belt TNOs have $q>30$ au (the solid black curve). In fact, most of these objects have $q=35-37 \mathrm{au}$, as shown in Petit et al. (2011).

Similar to the classical belt population, the detached objects (blue markers) do not interact with Neptune and remain separated from the $q=30$ au curve. Defined to be objects with higher eccentricities, the blue markers are found above the green ones.

The SDOs, marked in yellow, can be found near the $q=30 \mathrm{au}$ curve. These are TNOs with Neptune-driven dynamics, which result in their movement along the $q=30$ au curve. The perihelion distance threshold at which objects cease to be affected strongly by Neptune perturbations is often cited to be around $q \approx 35-37$ au (Jewitt 1999; Lykawka \& Mukai 2007); 
Table 1

Barycentric Orbital Elements for the Set of TNOs Detected by DES and Considered in This Work

\begin{tabular}{|c|c|c|c|c|c|c|c|c|c|}
\hline TNO ID & Class & Res. & $a_{b}(\mathrm{au})$ & $e_{b}$ & $i_{b}(\mathrm{deg})$ & $\omega_{b}(\operatorname{deg})$ & $\Omega_{b}(\operatorname{deg})$ & $M_{b}(\mathrm{deg})$ & Epoch (JD) \\
\hline 2003 QC112 & inner centaur & $\ldots$ & $20.5004 \pm 0.0009$ & $0.27616 \pm 3 \times 10^{-5}$ & $18.245 \pm 0.0001$ & $22.194 \pm 0.009$ & $158.6556 \pm 0.0003$ & $191.708 \pm 0.009$ & 2456578.73 \\
\hline 2014 UU240 & resonant & $1: 1$ & $30.056 \pm 0.001$ & $0.048 \pm 0.003$ & $35.747 \pm 0.004$ & $74 \pm 3$ & $81.993 \pm 0.004$ & $236 \pm 3$ & 2456959.83 \\
\hline 2013 VX30 & resonant & $1: 1$ & $30.0871 \pm 0.0006$ & $0.08374 \pm 2 \times 10^{-5}$ & $31.25873 \pm 7 \times 10^{-5}$ & $215.49 \pm 0.02$ & $192.53852 \pm 8 \times 10^{-5}$ & $347.84 \pm 0.02$ & 2456567.79 \\
\hline (530664) 2011 SO277 & resonant & $1: 1$ & $30.1614 \pm 0.0005$ & $0.01185 \pm 8 \times 10^{-5}$ & $9.6386 \pm 0.0002$ & $117.7 \pm 0.3$ & $113.5271 \pm 0.0009$ & $148.1 \pm 0.5$ & 2456545.88 \\
\hline (309239) 2007 RW10 & outer centaur & $\ldots$ & $30.236 \pm 0.002$ & $0.30055 \pm 6 \times 10^{-5}$ & $36.1011 \pm 0.0001$ & $96.095 \pm 0.005$ & $187.03731 \pm 7 \times 10^{-5}$ & $61.472 \pm 0.005$ & 2456547.85 \\
\hline 2013 RD109 & outer centaur & $\cdots$ & $32.378 \pm 0.001$ & $0.08106 \pm 2 \times 10^{-5}$ & $11.1194 \pm 0.0001$ & $332.64 \pm 0.05$ & $16.6919 \pm 0.0001$ & $11.31 \pm 0.05$ & 2456537.77 \\
\hline 2014 UC225 & scattering & $\cdots$ & $34.7_{-0.1}^{+0.4}$ & $0.1 \pm 0.04$ & $4.942 \pm 0.02$ & $221 \pm 100$ & $139.7 \pm 0.2$ & $17 \pm 90$ & 2456951.73 \\
\hline 2013 RH109 & resonant & $3: 4$ & $36.38 \pm 0.005$ & $0.0725 \pm 0.0002$ & $14.8214 \pm 0.0003$ & $288.5 \pm 0.3$ & $204.394 \pm 0.001$ & $196.8 \pm 0.3$ & 2456543.59 \\
\hline 2013 RQ109 & resonant & $3: 4$ & $36.404 \pm 0.003$ & $0.1512 \pm 6 \times 10^{-5}$ & $14.5378 \pm 0.0001$ & $335.91 \pm 0.03$ & $70.5182 \pm 0.0006$ & $339.72 \pm 0.02$ & 2456547.89 \\
\hline 2014 TM95 & resonant & $3: 4$ & $36.487 \pm 0.002$ & $0.18664 \pm 6 \times 10^{-5}$ & $17.5579 \pm 0.0001$ & $263.3 \pm 0.01$ & $161.1436 \pm 0.0004$ & $328.744 \pm 0.008$ & 2456569.69 \\
\hline 2013 SH102 & classical belt & $\ldots$ & $37.902 \pm 0.003$ & $0.0528 \pm 0.0002$ & $19.3819 \pm 0.0003$ & $83.07 \pm 0.07$ & $180.22736 \pm 2 \times 10^{-5}$ & $93.4 \pm 0.1$ & 2456565.66 \\
\hline 2013 RG109 & classical belt & $\cdots$ & $38.241 \pm 0.003$ & $0.0904 \pm 0.0002$ & $22.7219 \pm 0.0002$ & $59.72 \pm 0.04$ & $22.6841 \pm 0.0001$ & $298.16 \pm 0.02$ & 2456537.86 \\
\hline 2014 RH70 & classical belt & $\cdots$ & $38.251 \pm 0.008$ & $0.1224 \pm 0.0002$ & $27.60542 \pm 5 \times 10^{-5}$ & $244.72 \pm 0.06$ & $8.0072 \pm 0.0004$ & $39.53 \pm 0.04$ & 2456904.6 \\
\hline
\end{tabular}

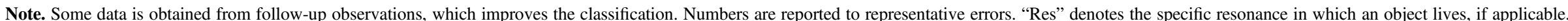

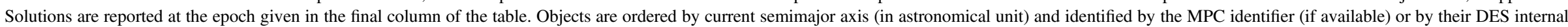
identifier if an MPC designation does not exist. Angles $(i, \omega, \Omega$, and $M)$ are given in degrees.

(This table is available in its entirety in machine-readable form.) 


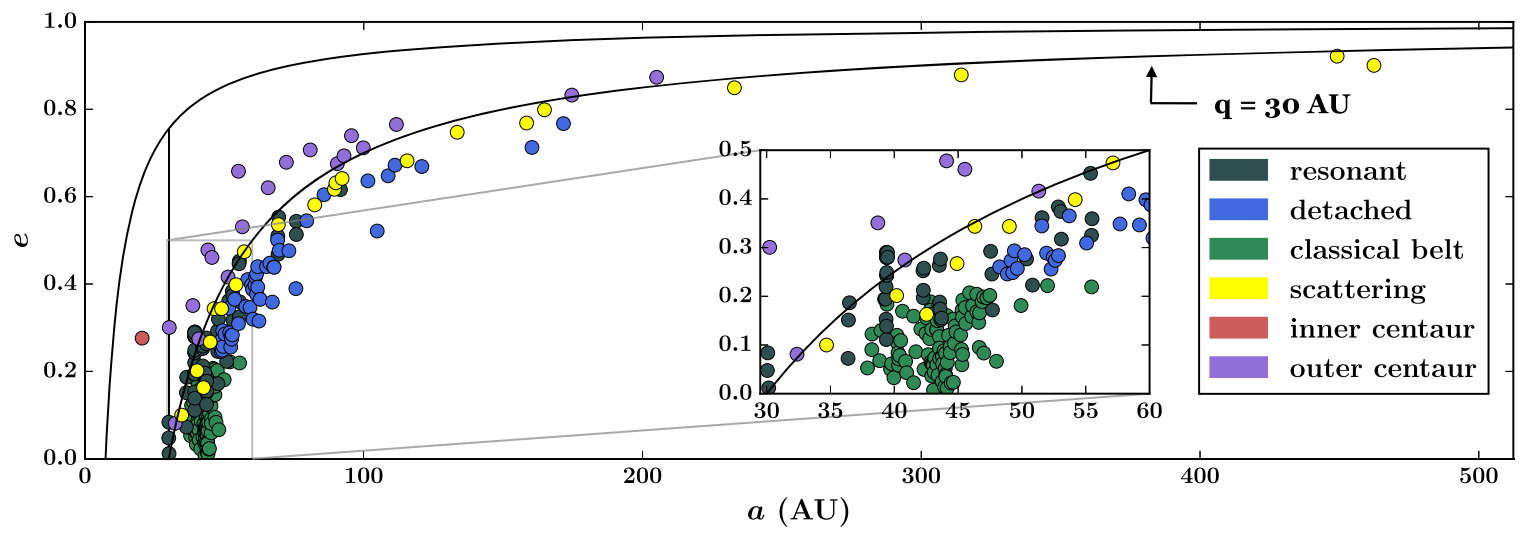

Figure 8. DES TNOs on the semimajor axis-eccentricity plane, with colored markers indicating the different dynamical classes into which objects have been classified. The black solid curves correspond to constant perihelion distances, with $q=7.35$ au and $q=30$ au (top to bottom); detections are biased toward objects whose current distances are closer, leading the envelope of the largest density of discovered objects to have a rough outer limit at around $q=35-36$ au. A companion plot that denotes the approximate region of each dynamical class is found in Figure 1. The inset zooms in on the $a \in[30,60]$ au, $e \in[0,0.5]$ region of the outer solar system. The orbital elements of the objects are plotted at the epoch reported in Table 1.

however, this boundary is actually dependent on semimajor axis (Duncan et al. 1987). Since a TNO's orbital energy scales as $1 / a$, at a fixed perihelion distance, larger semimajor axis objects are more strongly affected by energy kicks from Neptune and thus experience greater orbital evolution.

In the inset, it is possible to note objects marked with dark gray markers; these are the resonant and resonant candidate objects. These TNOs can be found in any region of the phase space, as their location is determined by their semimajor axis alone. For example, in the inset, it is easy to spot the three DES Neptune trojans at the $1: 1$ resonance at $a=30 \mathrm{au}$. A more detailed discussion of the resonant TNOs and a plot of the corresponding $a-e$ plane (Figure 10) are presented in the following section.

\subsection{Resonant Population}

The current DES TNO sample contains 48 resonant objects, with an additional 7 resonant candidates, as shown in Figure 9. In this plot, we present the results of our resonance classification algorithm for the entire DES sample. The histogram displays the percentage of time spent in resonance by each TNO.

To compute this value, we first find the fraction of time each of the 10 clones spends in a resonance during the integration time. Sometimes, a clone may visit more than one resonance during the integration; in this case, we take the longest time spent in one resonance. Next, we average over all of the 10 clones, and arrive at the percentage of time spent in resonance by each TNO.

The result is shown in the histogram in Figure 9. Note that there are two peaks of objects-nonresonant TNOs, with $0 \%$ of time spent in resonance, and securely resonant TNOs, with greater than $95 \%$ of time spent in resonance. There are relatively few TNOs in the middle region. This seems to indicate that our resonance-finding algorithm is able to clearly distinguish between the resonant and nonresonant cases, and does not present a large number of semiresonant objects.

In reality, objects could indeed be semiresonant: over long timescales, objects may transition in and out of resonance. The integration times under consideration here, however, are short, and we expect objects to either be resonant or not on these timescales.

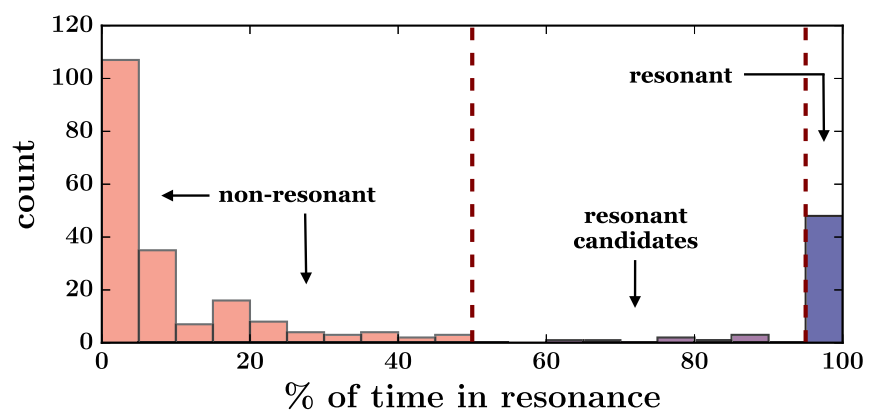

Figure 9. Histogram of the percent of time spent in resonance for all of the known DES TNOs. For each object, we compute the percent of time each of its clones spends in resonance and average over all 10 clones to find the total resonance percentage. Most objects are securely nonresonant (the $0 \%$ bin). In between the two dashed vertical lines are a few resonant candidates $(50 \%-$ $95 \%$ ), and to the right of the line at $95 \%$ are the resonant objects: those with clones that are in resonance for the full integration time.

In this work, we choose to identify TNOs that are resonant greater than $50 \%$ of the time, but less than $95 \%$ of the time, as resonant candidates. The location of the two thresholds is rather arbitrary, but Figure 9 clearly shows that any reasonable choice will produce quantitatively similar results. The candidate resonant TNOs spend the majority of their time in resonance; as their orbits improve with further observations, it is possible that these TNOs will become securely resonant TNOs.

The DES resonant TNOs populate 15 resonances, ranging from the short period Neptune trojans at the 1:1 resonance to the long period 3:16 TNO candidate. A bar plot of the populated resonances is shown in the bottom panel of Figure 10. The bottom left panel presents all instances of each $p: q$ resonance, sorted by increasing orbital period, from left to right. The blue bars represent the securely resonant objects, and the hatched purple bars show the much smaller population of resonant candidates. The bar plot in the bottom right panel summarizes this data.

The top panel displays the resonant objects on the $a-e$ plane. Note that each resonance is found at a constant semimajor axis as indicated by the dashed vertical lines; as a result, each resonance is reminiscent of beads on a string. Each of these resonances corresponds directly to a bar in the bottom left 

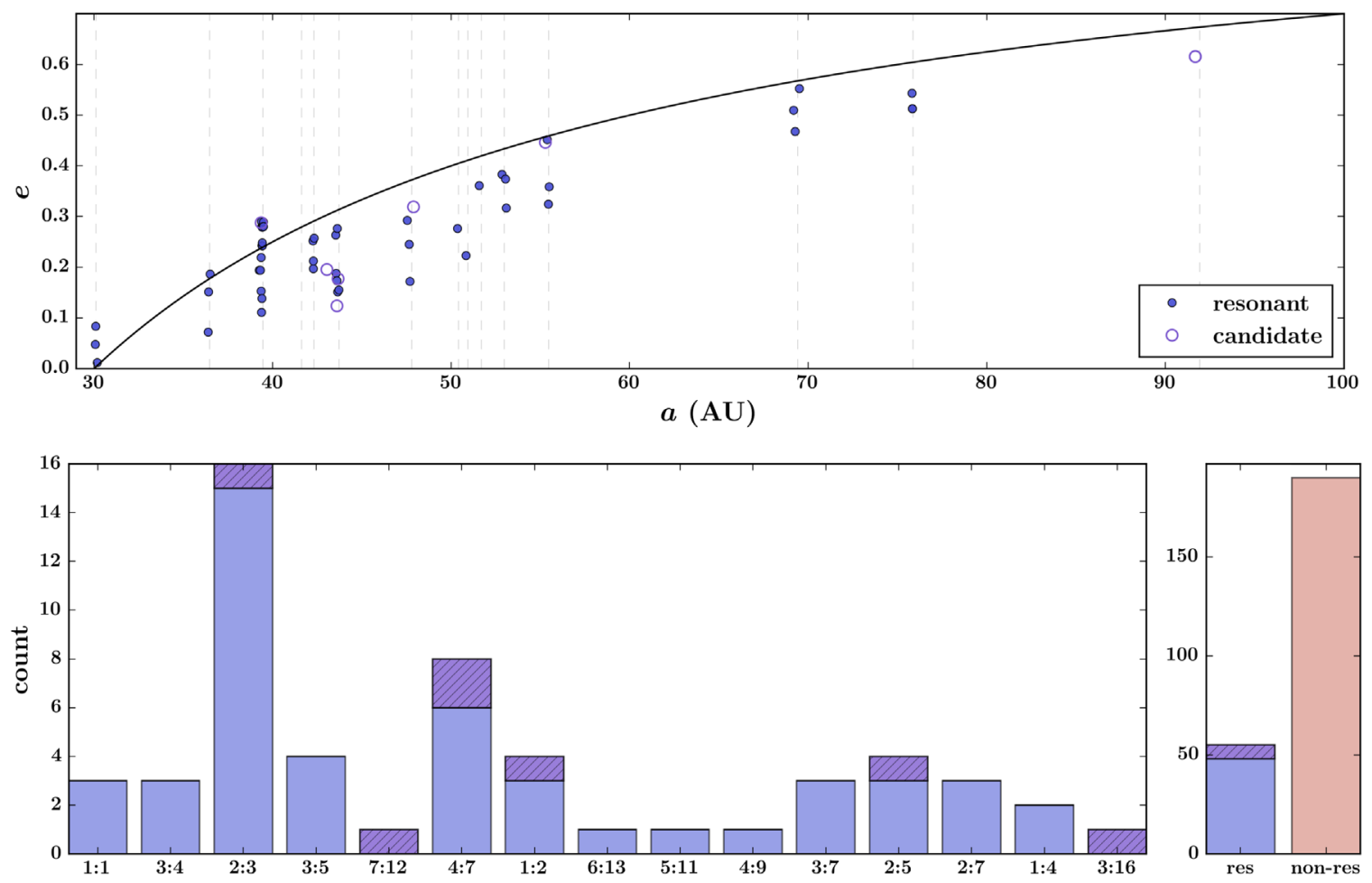

Figure 10. Top panel visualizes the resonant TNOs on the $a-e$ plane, while the bottom left panel presents the distribution of resonances for the resonant TNOs. All of the resonant objects are in resonance with Neptune. The most populated resonance is the 2:3 (the Plutinos), and there are a number of higher order resonances, such as the 2:7 or the 6:13. The bottom right panel shows the number of resonant objects as compared to the number of nonresonant TNOs in the DES data. The blue bars represent securely resonant objects, while the purple bars are the resonant candidates. The bottom left bar plot sorts the resonances by period; this allows for easy comparison between the bottom and top panels. For instance, the left three objects in both plots are the three Neptune trojans, and the rightmost TNO in both plots is a 3:16 resonant candidate. The orbital elements of the objects are plotted at the epoch reported in Table 1 .

panel. For example, note the three Neptune trojans on the left in both plots, next the three objects in the 3:4 resonance, and so on.

From this analysis, we see that the resonant TNOs make up a significant portion of the DES data set, representing about onefifth of the objects. The most populated resonances are the Plutinos, at the 2:3 resonance, but there are a number of higher order resonances in the sample as well.

\section{Discussion}

In this work, we introduce an updated classification algorithm for the trans-Neptunian region of the solar system. Our classification scheme is fundamentally consistent with the previous classification schemes laid out in Elliot et al. (2005) and Gladman et al. (2008). Similarly to Elliot et al. (2005), which used detections from the Deep Ecliptic Survey, we classify a uniformly derived sample of Kuiper Belt objects: all objects were detected so far in the DES data, many of which are previously undiscovered objects.

Our new resonance-finding tool allows for the automated identification of resonances by using numerical integrations of TNOs, and uses an hierarchical determination of regions where resonance angles librate to identify KBOs in true resonance. Through this method, we classify the current collection of objects detected by the DES and present a summary of the results. Our classification scheme yields 1 inner centaur, 19 outer centaurs, 21 SDOs, 47 detached TNOs, 48 securely resonant objects, 7 resonant candidates, and 97 classical belt objects.
It is important to note that our classification algorithm is only as good as the certainty of the TNO orbits. Although a poorly constrained orbit can result in a misclassification in any of the categories, the most sensitive boundary is that for the resonant classification. If the semimajor axis error for a TNO is several astronomical units or more-greater than a typical resonance width - then the spread in the initial orbit of the clones will result in overall nonresonant behavior for the TNO. In this situation, the object may be classified as scattering or as a classical belt/detached TNO, depending on its perihelion distance. In reality, however, the TNO could actually be in a resonance, but the wide range of possible semimajor axes $a$ (due to large uncertainties) prevents us from making a secure classification.

On the other hand, classifying an object as a securely resonant TNO is an indication that it has a well-defined orbit with small errors, and further observations of the object are unlikely to change the classification. That is, general improvement of the orbit certainties for the TNOs could potentially increase the number of objects in the resonance class, and decrease the number of objects in the other classes.

We expect the coming years to witness a substantial increase in the numbers of TNOs detected by DES as the remaining data is analyzed (e.g., Bernardinelli et al. 2019). Once the additional objects are classified and combined with the current data set, we plan to conduct a suite of population-wide analyses of the TNOs. In combination with the DES survey simulator, such future work will reveal the structure of this distant region and allow for the testing of formation hypotheses of the outer solar system. 
This material is based upon work supported by the National Aeronautics and Space Administration under grant No. NNX17AF21G issued through the SSO Planetary Astronomy Program. J.C.B, L.M., and S.J.H are supported by the NSF Graduate Research Fellowship grant No. DGE 1256260.

This work included the observations obtained at the MDM Observatory, operated by Dartmouth College, Columbia University, Ohio State University, Ohio University, and the University of Michigan.

Funding for the DES Projects has been provided by the U.S. Department of Energy, the U.S. National Science Foundation, the Ministry of Science and Education of Spain, the Science and Technology Facilities Council of the United Kingdom, the Higher Education Funding Council for England, the National Center for Supercomputing Applications at the University of Illinois at Urbana-Champaign, the Kavli Institute of Cosmological Physics at the University of Chicago, the Center for Cosmology and Astro-Particle Physics at the Ohio State University, the Mitchell Institute for Fundamental Physics and Astronomy at Texas A\&M University, Financiadora de Estudos e Projetos, Fundação Carlos Chagas Filho de Amparo à Pesquisa do Estado do Rio de Janeiro, Conselho Nacional de Desenvolvimento Científico e Tecnológico and the Ministério da Ciência, Tecnologia e Inovação, the Deutsche Forschungsgemeinschaft and the Collaborating Institutions in the Dark Energy Survey.

The Collaborating Institutions are Argonne National Laboratory, the University of California at Santa Cruz, the University of Cambridge, Centro de Investigaciones Energéticas, Medioambientales y Tecnológicas-Madrid, the University of Chicago, University College London, the DES-Brazil Consortium, the University of Edinburgh, the Eidgenössische Technische Hochschule (ETH) Zürich, Fermi National Accelerator Laboratory, the University of Illinois at UrbanaChampaign, the Institut de Ciències de l'Espai (IEEC/CSIC), the Institut de Física d'Altes Energies, Lawrence Berkeley National Laboratory, the Ludwig-Maximilians Universität München and the associated Excellence Cluster Universe, the University of Michigan, the National Optical Astronomy Observatory, the University of Nottingham, The Ohio State University, the University of Pennsylvania, the University of Portsmouth, SLAC National Accelerator Laboratory, Stanford University, the University of Sussex, Texas A\& M University, and the OzDES Membership Consortium.

Based in part on observations at Cerro Tololo InterAmerican Observatory, National Optical Astronomy Observatory, which is operated by the Association of Universities for Research in Astronomy (AURA) under a cooperative agreement with the National Science Foundation.

The DES data management system is supported by the National Science Foundation under grant Nos. AST-1138766 and AST-1536171. The DES participants from Spanish institutions are partially supported by MINECO under grants AYA2015-71825, ESP2015-66861, FPA2015-68048, SEV2016-0588, SEV-2016-0597, and MDM-2015-0509, some of which include ERDF funds from the European Union. IFAE is partially funded by the CERCA program of the Generalitat de Catalunya. Research leading to these results has received funding from the European Research Council under the European Union's Seventh Framework Program (FP7/20072013) including ERC grant agreements 240672, 291329, and 306478. We acknowledge support from the Brazilian Instituto
Nacional de Ciência e Tecnologia (INCT) e-Universe (CNPq grant 465376/2014-2).

This manuscript has been authored by Fermi Research Alliance, LLC under Contract No. DE-AC02-07CH11359 with the U.S. Department of Energy, Office of Science, Office of High Energy Physics. The United States Government retains and the publisher, by accepting the article for publication, acknowledges that the United States Government retains a nonexclusive, paid-up, irrevocable, world-wide license to publish or reproduce the published form of this manuscript, or allow others to do so, for United States Government purposes.

\section{ORCID iDs}

T. Khain (D) https://orcid.org/0000-0001-7721-6457

J. C. Becker (1) https://orcid.org/0000-0002-7733-4522

Hsing Wen Lin (林省文) (1) https://orcid.org/0000-00017737-6784

D. W. Gerdes (iD https://orcid.org/0000-0001-6942-2736

F. C. Adams (iD https://orcid.org/0000-0002-8167-1767

P. Bernardinelli (i) https://orcid.org/0000-0003-0743-9422

K. Franson (1) https://orcid.org/0000-0002-8906-2835

L. Markwardt (ib https://orcid.org/0000-0002-2486-1118

S. Hamilton (ib https://orcid.org/0000-0002-6126-8487

M. Sako (i) https://orcid.org/0000-0003-2764-7093

D. Brooks (i) https://orcid.org/0000-0002-8458-5047

D. L. Burke (iD https://orcid.org/0000-0003-1866-1950

M. Carrasco Kind (iD https://orcid.org/0000-0002-4802-3194

J. García-Bellido (iD https://orcid.org/0000-0002-9370-8360

D. Gruen (ib https://orcid.org/0000-0003-3270-7644

R. A. Gruendl (iD https://orcid.org/0000-0002-4588-6517

D. L. Hollowood (i) https://orcid.org/0000-0002-9369-4157

J. L. Marshall (iD https://orcid.org/0000-0003-0710-9474

R. Miquel (i) https://orcid.org/0000-0002-6610-4836

E. Sanchez (iD https://orcid.org/0000-0002-9646-8198

M. Smith (i) https://orcid.org/0000-0002-3321-1432

G. Tarle (D) https://orcid.org/0000-0003-1704-0781

A. R. Walker (1) https://orcid.org/0000-0002-7123-8943

\section{References}

Adams, E. R., Gulbis, A. A. S., Elliot, J. L., et al. 2014, AJ, 148, 55 Bannister, M. T., Gladman, B. J., Kavelaars, J. J., et al. 2018, ApJS, 236, 18 Batygin, K., Brown, M. E., \& Betts, H. 2012, ApJL, 744, L3 Becker, J. C., Khain, T., Hamilton, S. J., et al. 2018, AJ, 156, 81 Bernardinelli, P. H., Bernstein, G. M., Sako, M., et al. 2019, arXiv:1909.01478 Bernstein, G., \& Khushalani, B. 2000, AJ, 120, 3323

Bernstein, J. P., Kessler, R., Kuhlmann, S., et al. 2012, ApJ, 753, 152 Chen, Y.-T., Lin, H. W., Holman, M. J., et al. 2016, ApJL, 827, L24

Dark Energy Survey Collaboration, Abbott, T., Abdalla, F. B., et al. 2016, MNRAS, 460, 1270

Duncan, M., Quinn, T., \& Tremaine, S. 1987, AJ, 94, 1330

Elliot, J. L., Kern, S. D., Clancy, K. B., et al. 2005, AJ, 129, 1117

Flaugher, B., Diehl, H. T., Honscheid, K., et al. 2015, AJ, 150, 150

Gerdes, D. W., Jennings, R. J., Bernstein, G. M., et al. 2016, AJ, 151, 39

Gerdes, D. W., Sako, M., Hamilton, S., et al. 2017, ApJL, 839, L15

Gladman, B., Kavelaars, J., Petit, J. M., et al. 2009, ApJL, 697, L91

Gladman, B., Marsden, B. G., \& Vanlaerhoven, C. 2008, in Nomenclature in the Outer Solar System, ed. M. A. Barucci et al. (Tucson, AZ: Univ. Arizona Press), 43

Horner, J., Evans, N. W., \& Bailey, M. E. 2004, MNRAS, 354, 798 Jewitt, D. 1999, AREPS, 27, 287

Khain, T., Becker, J. C., Adams, F. C., et al. 2018, AJ, 156, 273

Kowal, C. T., Liller, W., \& Marsden, B. G. 1979, in IAU Symp. 81, Dynamics of the Solar System, ed. R. L. Duncombe (Dordrecht: D. Reidel), 245

Lan, L., \& Malhotra, R. 2019, CeMDA, 131, 39

Lawler, S. M., Kavelaars, J. J., Alexandersen, M., et al. 2018, FrASS, 5, 14 
Lin, W., Gerdes, D. W., Hamilton, S. J., et al. 2019, Icar, 321, 426

Lykawka, P. S., \& Mukai, T. 2007, Icar, 189, 213

Morbidelli, A., Emel'yanenko, V. V., \& Levison, H. F. 2004, MNRAS, 355,935

Nesvorný, D. 2011, ApJL, 742, L22

Petit, J.-M., Kavelaars, J. J., Gladman, B. J., et al. 2011, AJ, 142, 131

Schwamb, M. E., Brown, M. E., Rabinowitz, D. L., \& Ragozzine, D. 2010, ApJ, 720, 1691
Tiscareno, M. S., \& Malhotra, R. 2003, AJ, 126, 3122

Trujillo, C. A., Jewitt, D. C., \& Luu, J. X. 2001, AJ, 122, 457

Tsiganis, K., Gomes, R., Morbidelli, A., \& Levison, H. F. 2005, Natur, 435,459

Volk, K., \& Malhotra, R. 2017, AJ, 154, 62

Volk, K., Murray-Clay, R., Gladman, B., et al. 2016, AJ, 152, 23

Volk, K., Murray-Clay, R. A., Gladman, B. J., et al. 2018, AJ, 155, 260

Wang, X., \& Malhotra, R. 2017, AJ, 154, 20 